\title{
COVID-19-Cluster-Studie an einem Lehrkrankenhaus
}

Corona-Pandemie 2020 -- Autoren: A. von Freyburg, H. Hagedorn, B. Brücher, A. Schmidt, M. A. Scherer

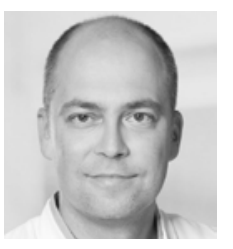

Alexander von Freyburg Leitung

Notaufnahme, HELIOS Amperklinikum Dachau

\section{INTERESSEN-} KONFLIKT

Die Autoren haben keinen Interessenskonflikte. Lediglich die Autoren sind verantwortlich für den Inhalt. Prof. Dr. Michael A Scherer ist Senior Editorial Board Mitglied von 4open von EDP Sciences und Prof. Dr. Björn Brücher ist hier Editor-in Chief. Teile der Ergebnisse dieses Manuskriptes wurden im kürzlich publizierten Manuskript, COVID-19: Pandemic Surgery Guidance', 4open 3(1), 1-19 [Referenz $13 \mathrm{im}$ hiesigen Manuskript] anonym aufgeführt und entsprechend dort erwähnt (Seite 11, Absatz 2, ff).

\section{UNTER-}

STÜTZUNG

Das Manuskript wurde unterstützt durch die Theodor-Billroth-Akademie ${ }^{\oplus}\left(\right.$ TBA $\left.^{\oplus}\right)$ und INCORE (International Consortium of Research Excellence) der TBA ${ }^{\oplus}$ Wir bedanken uns für die fortwährenden Diskussionen des Netzwerkes der TBA auf ihrer Web Group in Linkedln (>3.200 weltweite Mitglieder) wie auch dem kontinuierlichen Austausch über das wissenschaftliche Netzwerk ResearchGate, und dem Austausch mit Wissenschaft lern, was unser Denken und unsere Vorgehensweise angeregt hat wir danken jedem einzelnen.

\section{Zusammenfassung}

Anfang April 2020 wurde für ein Lehrkrankenhaus aufgrund eines COVID-19-Ausbruchs (Indexpatient und fünf infizierte Pflegekräfte) die Betriebsunterbrechung und Quarantäne gemäß $₫ 6$ Infektionsschutzgesetz angeordnet. Das komplette Personal (Ärzte, Pflegekräfte und nichtmedizinisches Personal [NMP]) wurde auf COVID-19 getestet. Die kumulierte Infektionsrate für NMP (1,6\%), Ärzte $(3,8 \%)$ und Pflegekräfte (9,7\%) stand im Zusammenhang mit Art und Umfang des COVID-19-Patientenkontaktes. Trotz COVID-19-Positivität von 34,8\% (46 von 132 Betten) ist bei Beachtung der Hygienevorschriften und strikter Patientenselektion ein risikoarmes Management des Krankenhausbetriebs bis zu einem gewissen Grad möglich. Allerdings ist aufgrund stiller Träger (silent carrier) ein COVID19-freies Klinikum nicht zu erwarten.

\section{Einleitung}

Die Weltgesundheitsorganisation (WHO) deklarierte am 10. Januar 2020 den Coronavirus-disease2019(COVID-19)-Epidemieausbruch in China [1] und am 11. März 2020 in ihrem Situation Report 51, dass für COVID-19 der Pandemiezustand besteht [2]. Am 19. März 2020 erließ das Bayerische Staatsministerium für Gesundheit und Pflege auf der Grundlage des Infektionsschutzgesetzes eine Allgemeinverfügung, in der „soweit medizinisch vertretbar bis auf weiteres alle planbaren Behandlungen zurückzustellen oder zu unterbrechen sind, um möglichst umfangreiche Kapazitäten für die Versorgung von COVID-19-Patienten freizumachen“ (BayMBI) [3]. Daraufhin erfolgten Anstrengungen, entsprechende strukturelle sowie personelle Voraussetzungen zu schaffen, um einem möglichen COVID19-Patientenaufkommen so gut wie möglich gewachsen zu sein.

\section{Ausgangspunkt}

Am Nachmittag des 3. April 2020 wurde am HELIOS Amperklinikum Dachau nach Erhalt von positiven COVID-19-Testergebnissen von fünf Mitarbeitern der Intermediate-Care(IMC)-Station ein Indexpa- tient ermittelt, der danach auf Normalstation verlegt worden war. Damit war ein Ausbruchsgeschehen nach $₫ 6$ Infektionsschutzgesetz und plötzlich eine sehr hohe Zahl von Kontaktpersonen der Kategorie 1 gegeben, sodass das Gesundheitsamt einen Shutdown des Krankenhauses verfügte und alle Mitarbeiter sowie sämtliche Patienten unter Quarantäne stellte.

Weiterhin wurde sofort die Entscheidung getroffen, dass sich das komplette Personal (Ärzte, Pflegekräfte und nichtmedizinisches Personal [NMP]) PCRTestungen auf COVID-19 zu unterziehen hatte, um sowohl symptomfreie stille Träger („silent carrier“) als auch infizierte Mitarbeiter zu identifizieren und rechtzeitig eine weitere Infektionsausbreitung zu unterbinden.

\section{Methodik}

Die COVID-19-Testung der Mitarbeiter erfolgte in zwei zeitlichen Serien (Anzahl Positivität):

1. zwischen 3. April 2020 16.30 Uhr und 5. April $202016.00 \mathrm{Uhr}(\mathrm{n}=1.170)$. Zeitgleich wurden die Mitarbeiter über die Quarantäne-Richtlinien, wie sie vom Gesundheitsamt vorgegeben waren, schriftlich informiert.

2. zwischen dem 8. und 9. April 2020 ( $n=953)$. Dazu wurden alle Mitarbeiter, die bei der ersten Untersuchung COVID-19-negativ waren, über E-Mail und Aushang informiert.

Aus der Mitternachts-Belegungsstatistik am 3. April $2020(\mathrm{n}=197)$ und 8. April $2020(\mathrm{n}=132)$ und der zum jeweiligen Datum bekannten Anzahl COVID19-positiver Patienten ( $\mathrm{n}=33$ bzw. $\mathrm{n}=46$ ) wurde die einfache Verhältniszahl der Krankenhaus-Bettenbelegung mit COVID-19-positiven und -negativen $\mathrm{Pa}$ tienten in Prozent als Surrogatparameter für das Ansteckungspotenzial angegeben.

Die Referenzzahl zur COVID-19-Infektion in der Bevölkerung wurde dem Zeit-online-Corona-Ticker entnommen [4], der am 3. April $2020 \mathrm{n}=457$ und am 8. April $2020 \mathrm{n}=555$ bestätigte Coronafälle im Landkreis Dachau mit seinen 154.544 Einwohnern auswies [5]. 
Die Auswertung hinsichtlich der Inzidenz von COVID-19-positiven Befunden stratifiziert nach Berufsgruppen wurde mit dem $\mathrm{Chi}^{2}$-Test für unverbundene Stichproben zweiseitig analysiert und als Nullhypothese wurde formuliert, dass der ausgeübte Beruf keinen Einfluss auf die Infektionszahlen hat [6].

\section{Ergebnisse}

Der Landkreis Dachau gehörte am 14. April 2020 mit 684 positiv getesteten Personen, entsprechend einem Anteil von 45,3 Infizierten/10.000 Einwohner (Infektionsrate $0,45 \%$ ), zu den stark betroffenen Landkreisen und Städten Deutschlands [4].

Abb. 1 verdeutlicht den zeitlichen Verlauf der Belastung des Krankenhauses mit COVID-19-Patienten zwischen dem 24. März und dem 14. April 2020. Mit zeitlicher Verzögerung zur Durchseuchung in der Normalbevölkerung ergab sich am 30. März 2020 ein rascher Anstieg der COVID-19-positiven Patienten in der Klinik mit einer Verdopplungszeit von 4,6 Tagen im Gegensatz zum Landkreis mit 10,2 Tagen. Am 14. April 2020 zeigte sich ein anderes, positives Bild: Die Verdopplungszeit im Landkreis war auf knapp 14 Tage angestiegen, die Zahl der hospitalisierten Patienten mit positivem COVID-19-Test konstant geblieben.

Der Bedarf an Beatmungsplätzen, die von COVID19-positiven Patienten beansprucht wurden, hatte am 3. April 2020 die volle Normalkapazität an Beatmungsplätzen $(\mathrm{n}=12)$ beansprucht, eine Ausweitung auf die vorbereiteten 20 Beatmungsplätze war nur in geringem Umfang (4 zusätzliche Betten) erforderlich. 10 COVID-19-Patienten verstarben trotz

\section{Abb. 1 COVID-19-positive Patienten, Allokation und Krankheitsverlauf}

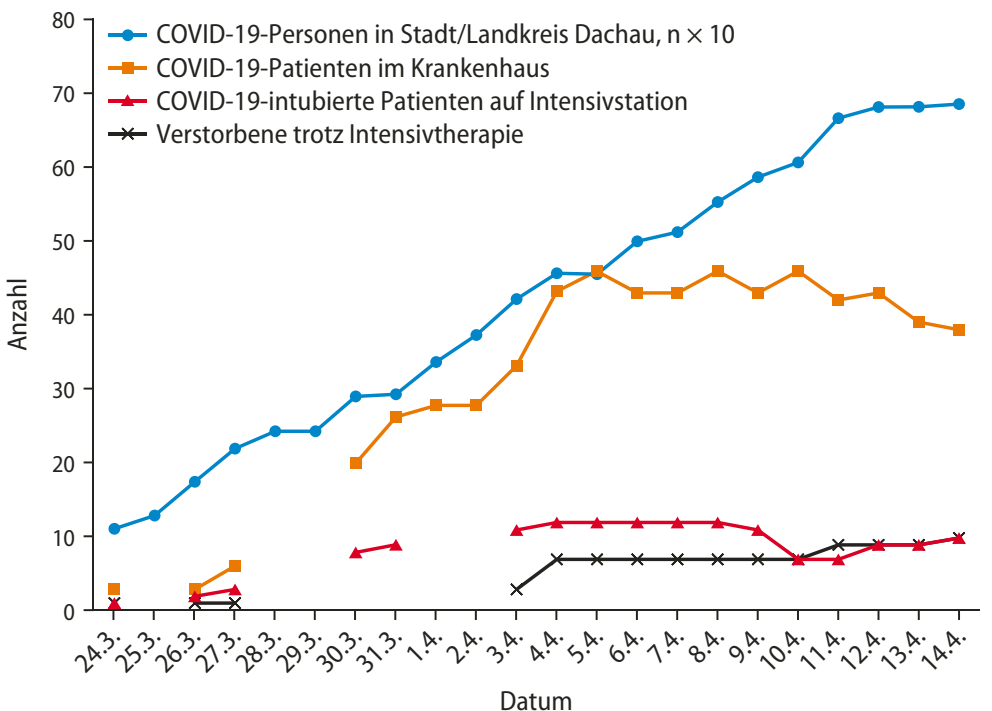

Intensivtherapie, die offizielle Statistik schreibt dem Landkreis 14 Todesfälle zu.

In der ersten COVID-19-Testserie (3. bis 5. April 2020) wurden 1.170 Mitarbeiter und in der zweiten Serie (8. bis 9. April 2020) 953 Mitarbeiter getestet. Der Nachweis von COVID-19-stillen Trägern („silent carrier") (Positivität) ergab für die erste Serie $\mathrm{n}=19(1,6 \%)$ und für die zweite Serie $\mathrm{n}=25(2,6 \%)$. Die Verteilung der betroffenen Personen auf die einzelnen Berufsgruppen ist in Tab. 1 dargestellt. Bezüglich Tätigkeit zeigte sich eine kumulierte Infektionsrate für NMP (1,6\%), Ärzte (3,8\%) und Pflege $(9,7 \%)$ als Zusammenhang mit Art und Umfang des COVID-19-Patientenkontaktes.

\section{Diskussion}

Das Lehrkrankenhaus hält normalerweise 435 Betten vor. Im Rahmen des Erweiterungsbaus und einer Kernsanierung unter laufendem Betrieb (Planungszeitraum von 5 Jahren) standen vor der Corona-Krise durchschnittlich 290 Betten im Haupthaus zur Verfügung. Mit dem Shut-down wurde die Belegung bis auf 132 Betten (45\%) zurückgefahren, wobei am 8. April 2020 jeder dritte Patient im Krankenhaus COVID-19-positiv getestet war.

\section{Frühe Analyse zeigt Transmissionswege auf}

In der frühen Analyse von 138 Patienten aus Wuhan im Zeitraum vom 1. bis 28. Januar 2020 [7] wurde eine Infektion im Krankenhaus beim Krankenhauspersonal ( $n=40,29 \%$ der Fälle) bzw. eine nosokomiale Infektion ( $\mathrm{n}=17,12,3 \%)$ als wesentlicher Transmissionsweg identifiziert. Die spätere Detailanalyse von 32.583 bestätigten COVID-19-Fällen in China ergab eine Anzahl von 1.496 (4,6\%) infizierten Krankenhausmitarbeitern [8].

In der weiteren Analyse hatte die hohe Gesamtzahl infizierter Krankenhausmitarbeiter - $\mathrm{n}=3.019$, 3,83\% bezogen auf die Gesamtzahl der Infektionsfälle $(n=82.623)$ - einen direkten Einfluss auf die Patientenversorgung und die Gesamtletalität [9]. In diesen Untersuchungen wurde jeweils die Anzahl infizierter Krankenhausmitarbeiter in Relation zur Gesamtzahl der erfassten Infizierten gesetzt, nicht zur Anzahl der beschäftigten Mitarbeiterinnen und Mitarbeiter.

\section{Wie hoch ist das Infektionsrisiko der Krankenhausmitarbeiter?}

Nach bestem Wissen stellt diese Untersuchung die erste zeitliche Längsschnittuntersuchung über das Gefährdungspotenzial bei Mitarbeiterinnen und Mitarbeitern im deutschen Gesundheitswesen dar. Bei Verwendung der gleichen Bezugsgrößen wie in den chinesischen Arbeiten, also erkrankten Mitarbeitern in Bezug zur Gesamtzahl der Erkrankten in 


\section{Abstract:}

On Mar 11th, 2020, the World Health Organization (WHO) stated in its Situation Report - 51 Coronavirus disease 2019 (COVID-19) as a pandemic. In early April 2020, a teaching hospital underwent shutdown and quarantine due to an outbreak of infection in accordance with Section 6 of the Infection Protection Act (index patient and 5 infected nursing staff). The complete staff (physicians, nurses and nonmedical personnel [NMP]) underwent COVID-19 testing within two phases: (1) between Apr 3rd and 5th, 2020 [n=1170], followed by (2) between Apr 8th and 9th, 2020 [n=953] with COVID-19 silent carrier positivity rates in accordance to testing phases of (1) $n=19$ (1.6\%) and (2) $n=25$

(2.6\%). The cumulative infection rate for NMP (1.6\%), doctors (3.8\%) and nurses (9.7\%) was connected to type and extent of COVID-19 patient contact. Despite COVID-19 positivity of $34.8 \%$ (46 of 132 beds), a risk-free management of hospital operation is possible to a certain extent if hygiene regulations and strict patient selection are followed. However, a COVID-19-free clinic cannot be expected due to silent carriers.

Literatur: springermedizin.de/mmw

Title:

COVID-19 cluster study at a teaching hospital

\section{Keywords:}

Hospital, healthcare workers, COVID-19, silent carrier, infection rate

Tab. 1 Stratifizierung nach Berufsgruppen, positivem COVID-19-Abstrich und kumulierter Infektionsrate

\begin{tabular}{|c|c|c|c|c|c|c|}
\hline Berufsgruppe & $\mathbf{n}_{\text {ges }}$ & $\mathrm{CV}+_{\text {prae }}$ & $\mathrm{CV}+{ }_{03.04}$ & $\mathrm{CV}+{ }_{08.04}$ & $\mathrm{CV}+{ }_{14.04}$ & kIR \% \\
\hline Bevölkerung & 154.544 & & 457 & 555 & 684 & 0,5 \\
\hline $\begin{array}{l}\text { Angestellte } \\
\text { Pflege } \\
\text { Ärzte } \\
\text { Physio/Ergo/Logo }\end{array}$ & $\begin{array}{r}505 \\
413 \\
208 \\
44\end{array}$ & $\begin{array}{l}1 \\
8 \\
3 \\
1\end{array}$ & $\begin{array}{r}3 \\
15 \\
3 \\
1\end{array}$ & $\begin{array}{r}4 \\
17 \\
2 \\
0\end{array}$ & & $\begin{array}{l}1,6(n=8) \\
9,7(n=40) \\
3,8(n=8) \\
4,5(n=2)\end{array}$ \\
\hline $\begin{array}{l}\text { Patienten 03.04. } \\
\text { Patienten 08.04. } \\
\text { Patienten 14.04. }\end{array}$ & $\begin{array}{l}197 \\
132 \\
203\end{array}$ & & 33 & 46 & 38 & $\begin{array}{l}16,7 \\
34,8 \\
18,7\end{array}$ \\
\hline
\end{tabular}

$n_{\text {ges }}=$ Gesamtzahl; $C V+_{\text {prae }}=$ Anzahl positiver COVID-19-Befund bis 2.4.2020; CV $+_{\text {Datum }}=$ Anzahl positiver COVID-19-Befund zum jeweiligen Testtag; $k I R=$ kumulierte Infektionsrate zum Stichtag 14.4.2020 in $n$ und $\%$.

einer Region, ergäbe sich für Dachau ein erheblicher Wert von $10,45 \%$ (58/555 am 8.4.2020). Die Infektionsrate für die Landkreisbevölkerung lag bei 0,46\%. Alle Mitarbeiter, die einen direkten $\mathrm{Pa}$ tientenkontakt hatten, zeigten demgegenüber eine deutlich erhöhte Infektionsrate, die im zweiseitigen $\mathrm{Chi}^{2}$-Test eine signifikante Risikoerhöhung der Pflege gegenüber allen anderen Berufsgruppen erreicht ( $\mathrm{p}<0,5, \mathrm{Chi}^{2}$-Wert 6,613).

Am 19. Februar 2020 [10] und 21. Februar 2020 [11] wurde der Nachweis geführt, dass klinisch und radiologisch asymptomatische Personen das Virus übertragen können. Die Viruslast ist dabei nasal höher als pharyngeal und sinkt tendenziell mit zunehmender Erkrankungsdauer [10]. Experimentell erzeugte, Virus-beladene Aerosole ergaben lebendige, infektiöse Viruspartikel während der dreistündigen Experimentdauer. SARS-CoV-2 hatten eine mediane Überlebensdauer von 5,6 Stunden auf Stahl und 6,8 Stunden auf Plastikoberflächen, sehr kleine Virusmengen konnten selbst nach 72 Stunden noch auf diesen Oberflächen nachgewiesen werden [12]. Die Infektion durch Aerosole ist der Hauptübertragungsweg [13].

\section{Vorsichtsmaßnahmen zeigen Wirkung}

Bei Einhaltung sämtlicher Hygienemaßnahmen und Etablierung einer Aufnahmeprozedur aus Temperaturmessung, Symptomscreening, Reise- und Sozialanamnese wird aus Singapur eine Null-Prozent-Rate nosokomialer Infektionen nach Behandlung von 147 Verdachtsfällen berichtet [14].

Die Strategie von möglichst umfassender Identifizierung von Infizierten, Isolierung und Nachverfolgung von Kontaktpersonen, rigorosem Screening bis hin zur vollständigen Testung und Einzelzimmerisolierung aller Patienten, die stationär aufgenommen werden, hat trotz steigender Infektionszahlen im Landkreis zu einer Stabilisierung der Zahl hospitalisierter und beatmeter Patienten beigetragen. Die wiederholte konsequente Testung aller COVID19-negativen Mitarbeiter und das Aufdecken und die Testung von Kontaktpersonen, sei es durch eine App oder anhand von Anamnesebögen, kann in bis zu $25 \%$ asymptomatische Träger identifizieren [15]. Im Operationssaal als Hochrisikobetrieb für eine Ansteckung des Krankenhauspersonals sind extensive Maßnahmen zur Selektion von asymptomatische Virusträgern $\mathrm{zu}$ fordern [13]. Hierzu wurde von einem internationalen Konsortium eine PandemieChirurgie-Anleitung etabliert und publiziert, die weltweit sowohl jetzt in der COVID-19-Krise als auch bei zukünftig zu erwartenden Pandemien nutzbar ist.

Trotz COVID-19-Positivität von 34,8\% (46 von 132 Betten) ist bei Beachtung der Hygienevorschriften und strikter Patientenselektion ein risikoarmes Management des Krankenhausbetriebs bis zu einem gewissen Grad möglich. Allerdings ist aufgrund stiller Träger („silent carrier“) ein COVID-19-freies Klinikum nicht zu erwarten.

\section{Autoren:}

\section{Alexander von Freyburg}

Leitung Notfaufnahme, HELIOS Amperklinikum Dachau

\section{Prof. Dr. med. Hjalmar Hagedorn}

Chefarzt Hals-, Nasen- und Ohrenheilkunde, HELIOS Amperklinikum Dachau

\section{Prof. Dr. med. Björn Brücher}

Theodor-Billroth-Academy ${ }^{\circledast}$, München, Deutschland - Sacramento CA, USA, INCORE, International Consortium of Research Excellence of the Theodor-BillrothAcademy ${ }^{\oplus}$, München, Deutschland - Sacramento, CA, USA und Chirurgische Klinik, Carl-Thiem-Klinikum, Cottbus

\section{PD Dr. Annette Schmidt}

Institut für Pharmakologie und Toxikologie der Bundeswehr, München

Prof. Dr. med. Michael A. Scherer (korrespondierender Autor)

Abteilung für Unfallchirurgie und Orthopädie,

HELIOS Amperklinikum Dachau,

Krankenhausstraße 15, D-85221 Dachau,

E-Mail michael.scherer@helios-gesundheit.de

und Theodor-Billroth-Academy ${ }^{\circledast}$, München, Deutschland -

Sacramento CA, USA 


\section{Literatur}

1. World Health Organization (WHO) (2020), Statement on the second meeting of the International Health Regulations (2005) Emergency Committee regarding the outbreak of novel coronavirus (2019-nCoV), Jan 30, 2020. Available from: https://www. who.int/news-room/detail/30-01-2020-statement-on-the-second-meeting-of-the-international-health-regulations(2005)-emergency-committee-regarding-the-outbreak-ofnovel-coronavirus-(2019-ncov). (Accessed March 2020).

2. World Health Organization (WHO) (2020), Coronavirus disease 2019 (COVID-19) Situation Report -51. Available from URL: https://www.who.int/docs/default-source/coronaviruse/situation-reports/20200311-sitrep-51-covid-19. pdf?sfvrsn=1ba62e57_10. (Accessed March 2020).

3. Bayerisches Ministerialblatt BayMBI $2020 \mathrm{Nr} .151$ vom 25.03.2020 zum 19.03.20, Az G24-K9000-2020/125.

4. Coronarvirus Karte für Deutschland (2020), Wie sich das Coronavirus in Ihrer Region ausbreitet, Die Zeit, Apr 04, 2020. Available from: https://www.zeit.de/wissen/gesundheit/coronavirus-echtzeit-karte-deutschland-landkreise-infektionen-ausbreitung. (Access April 2020).

5. Landratsamt Dachau (2020), Gemeinden- und Anwohnerzahlen. Available from: https://www.landratsamt-dachau.de/ landkreis-kultur-tourismus/landkreis/gemeinden-einwohnerzahlen. (Access Apr 2020).

6. Sachs L (1968), Statistische Anwendungsmethoden, SpringerVerlag - Berlin, Heidelberg, New York. ISBN 978-3-662-057452.

7. Wang D, Hu B, Hu C, Zhu F, Liu X, Zhang J, Wang B, Xiang H, Cheng Z, Xiong Y, Zhao Y, Li Y, Wang X, Peng Z. (2020), Clinical characteristics of 138 hospitalized patients with 2019 novel coronavirus infected pneumonia in Wuhan, China, JAMA 2020 Feb 7. D0l: $10.1001 /$ jama.2020.1585.

8. Pan A, Liu L, Wang C, Guo H, Hao X, Wang Q, Huang J, He NYu H, Lin X, Wei S, Wu T (2020), Association of public Health interventions with the epidemiology of the COVID-19 outbreak in Wuhan, China, JAMA Apr 10, 2020. D01:10.001/jama.2020.6130.

9. Wang Y, Wang Y, Chen Y, Qin Q (2020), Unique epidemiological and clinical features of the emerging 2019 novel coronavirus pneumonia (COVID-19) implicate special control measures, J Med Virol Mar 05, 2020 D0l: 10.1002/jmv.25748.

10. Wilder-Smith A, Chiew CJ, Lee VJ (2020), Can we contain the COVID-19 outbreak with the same measures as for SARS? Lancet Infect Dis Mar 05, 2020, pii: S1473-3099(20)30129-8. D0I: 10.1016/S1473-3099(20)30129-8.

11. Bai Y, Yao L, Wei T, Tian F, Jin DY, Chen L, Wang M (2020), Presumed Asymptomatic Carrier Transmission of COVID-19, JAMA Feb 21, 2020. D01: 10.1001/jama. 2020.2565.

12. Doremalen N v, Morris DH, Holbrook MG, Gamble A, Williamson BN, Tamin A, Harcourt JL, Thornburg NJ, Gerber SI, Lloyd-Smith J0, Wit E de, Munster VJ (2020), Aerosol ans surface stability of SARS-COV-2 as compare dto SARS-CoV-1 Correspondence, NEJM March 17, 2020. D01:10.1056/NEJMc2004973.

13. Brücher BLDM, Nigri G, Tinelli A, Lapeña JFF Jr., Espin-Basany E, Macri P, Matevossian E, Ralon S, Perkins R, Lück R, Kube R, da Costa JSC, Mintz Y, Tez M, Allert S, Sökmen S, Spychala A, Zilberstein B, Marusch F, Kermansaravi M, Kycler W, Vicente D, Scherer MA, Rivkind A, Elias N, Wallner G, Roviello F, Santos LL, Araujo RJC Jr., Szold A, Oleas R, Rupnik MS, Salber J, Jamall IS, Engel A (2020), COVID-19: Pandemic surgery guidance, 4open 3(1), 1-19. DOl: 10.1051/fopen/2020002.

14. Lee IK, Wang CC, Lin MC, Kung CT, Lan KC, Lee CT (2020), Effective strategies to prevent coronavirus disease-2019 (COVID-19) outbreak in hospital, J Hosp Infect Mar 3, 2020, pii: S01956701(20)30098-0. D0l: 10.1016/j.jhin.2020.02.022.

15. Ng Y, Li Z, Chua YX, Chaw WL, Zhao Z, Er B, Pung R, Chiew CJ, Lye DC, Heng D, Lee VII (2020), Evaluation of the Effectiveness of Surveillance and Containment Measures for the First $100 \mathrm{~Pa}$ tients with COVID-19 in Singapor, MMWR Morb Mortal Wkly Rep 69(11), 307-311. D0I: 10.15585/mmwr.mm6911e1. 